\title{
Biocombustibles: seguridad energética y sostenibilidad. Conceptualización académica e implementación en Colombia
}

\author{
Carlos Ariel Ramírez Triana \\ carlosar@poligran.edu.co \\ carlos.ramirez.triana@gmail.com \\ Politécnico Grancolombiano
}

\begin{abstract}
Reseña del autor
El autor es economista y máster en Economía, con énfasis en Industria y Tecnología, de la Universidad Nacional de Colombia. Actualmente, adelanta sus estudios de doctorado en Macquarie Graduate School of Management (MGSM) en la Macquarie University (MU), Australia. Es profesorinvestigador del Politécnico Grancolombiano en el área de Economía y tutor de Microeconomía en la Facultad de Economía y Finanzas de mu. Se encuentra vinculado al Centro de Investigación Colombia Regional y Empresarial del Politécnico Grancolombiano.
\end{abstract}

\section{Resumen}

Este artículo hace un recorrido por la literatura académica para definir y clasificar los biocombustibles, y establecer su importancia entre las alternativas energéticas renovables. Asimismo, explora los conceptos de desarrollo sostenible y seguridad energética, planteados por el Gobierno nacional como justificación de esta industria.

Una vez aceptados estos argumentos para el caso colombiano, se presenta una revisión histórica del proceso de implementación de biocombustibles, para entender el estado tecnológico de esta industria y su situación frente a un modelo ideal.

\section{Palabras clave}

Biocombustibles, energía renovable, biomasa, sostenibilidad.

\author{
Abstract \\ This paper presents an academic literature \\ review defining and classifying biofuels, \\ and establishing their importance among \\ renewable energy alternatives. In the \\ same way, it explores both sustainable \\ development and energy security concepts, \\ which are set by national government as the \\ main justification for this industry. \\ Once these arguments are accepted, \\ there is a historic review of the biofuel \\ industry implementation, in order to \\ understand its technological status and \\ contrast with an ideal model.
}

\section{Keywords}

Biofuels, Renewable Energy, Biomass,

Sustainability. 


\section{Introducción}

Desde el 2005, en Colombia se ha experimentado la implementación de alcohol carburante y se ha hablado de un plan que ya está en marcha, e incluye grandes plantaciones de palma de aceite para la producción de biodiesel. Dentro de los planes del Gobierno colombiano, como consta en el documento Conpes número 3510 , se ha desarrollado una política para promover la producción de biocombustibles -BCs- en Colombia (DNP, 2008); pero se le ha otorgado un papel casi mesiánico al desarrollo bioenergético del país y, si bien hay una serie de consecuencias positivas que se pueden derivar de su implementación, ciertamente existen riesgos muy altos asociados a este proyecto.

A medida que esta industria avanza, también lo hace su conceptualización. Esta situación da lugar a diferentes aproximaciones e interpretaciones y, en consecuencia, a estrategias reflejadas en las políticas energéticas adoptadas por un país. Si bien en un principio, los BCs fueron entendidos como un componente energético típico de economías en desarrollo o subdesarrolladas, en la actualidad son concebidos por algunos autores (Dale, 2007; Mathews, 2007a) como una moderna y posible alternativa ambientalmente amigable, frente a los hidrocarburos usados exclusivamente para locomoción.
En Colombia resulta importante entender, no solo qué son los BCs, sino hasta dónde ha llegado el avance de éstos y sus implicaciones en sostenibilidad. Si se ignora esto, se corre el riesgo de dinamizar el sector agrícola sólo en el corto plazo, particularmente en los cultivos de caña y palma aceitera, para cubrir la demanda interna; pero en el largo plazo, la industria bioenergética puede estancarse, si no se siguen los parámetros dictados por el consenso internacional.

El Gobierno nacional argumenta que el desarrollo de esta industria es necesario, porque contribuye a la seguridad energética, disminuye los impactos ambientales de los combustibles fósiles y estimula el sector rural. Sin embargo, a partir de lo anterior, surgen varios interrogantes: ¿qué son y qué clases de BCs hay en el mercado?, ¿¿cuáles se producen en Colombia? y finalmente, ¿'su forma de implementación es consecuente con los objetivos planteados?

Para responder estas preguntas, se exponen elementos básicos para entender la industria de BCs en general. Allíse presentan las definiciones generales de los BCs, y también se plantea breve ejercicio taxonómico, que obedece a la naturaleza física y los procesos tecnológicos de cuatro generaciones de BCs.

Asimismo, se ahonda en las definiciones de los motores que impulsan esta industria: sostenibilidad, que en 
este caso abarca el desarrollo agrícola y el aumento de la calidad ambiental; y seguridad energética, con sus implicaciones socioeconómicas.

En la tercera sección se da paso a la exposición de la situación actual del país, en torno a este tema. Primero se muestran los recursos energéticos disponibles en el país, y posteriormente, las características generales de la industria de всs en Colombia. Finalmente, se contrasta dicho funcionamiento a la luz de la sostenibilidad y seguridad energética. Sobre la base anterior, la conclusiones exponen la validez de los argumentos que se esgrimen para defender la necesidad de producción de los BCs o el acierto en la forma en la que éstos se implementan en Colombia.

\section{Definiciones básicas: biocombustibles, sostenibilidad y seguridad energética}

En este artículo se expondrá la posición oficial del Gobierno colombiano frente a la política de BCs y se presentará un breve paralelo con lo desarrollado en torno al mismo tema, en el ámbito mundial. Antes de entrar en detalle, es preciso esclarecer la terminología que será usada, tal cual se presenta en la esfera académica. Dado esto, en la presente sección se describirán y contextualizarán brevemente los términos BCs, desarrollo sostenible y seguridad energética.

\section{BCS: definición y clasificación}

La primera ley de la termodinámica ${ }^{1}$ explica cómo la energía a la que el consumidor final se enfrenta es la consecuencia de una serie transformaciones energéticas anteriores; de forma que, por ejemplo, la luz que usada para leer este artículo es el resultado de la transformación de energía eléctrica en lumínica, que a su vez fue precedida por la transformación de energía potencial -acumulada en un embalse- en energía eléctrica, liberada a través de una turbina hidroeléctrica.

Así como el anterior, es posible mencionar un gran número de ejemplos de transformación -no creaciónde energía, y la producción de BCs es uno más de ellos. La bioenergía es una forma indirecta de aprovechamiento de la energía solar, proceso en el que, a través de la fotosíntesis, se mezcla el dióxido de carbono $-\mathrm{CO}_{2}^{-}$, agua $-\mathrm{H}_{2} \mathrm{O}-$, luz y clorofila, para obtener un compuesto orgánico $-\mathrm{CH}_{2} \mathrm{O}-$, denominado carbohidrato, y oxígeno $-\mathrm{O}_{2}-$.

El principio de conservación de energía fue enunciado en 1824 por el francés Nicolás Carnot: En un sistema aislado, la energía no se crea ni se destruye, sólo se transforma. 
Los carbohidratos son la forma biológica primaria de almacenamiento y consumo de energía. En las plantas, estos compuestos adoptan varias formas, entre las que se encuentran los azúcares y los almidones; mientras que en los animales, por lo general, se transforman en grasas o lípidos. De cualquier manera, antes de que la energía contenida en este material esté disponible, se requieren algunos procesos de transformación -cultivo, extracción, fermentación, esterificación, por mencionar algunos ejemplos-,y dichos procesos constituyen la base de la industria bioenergética.

Este artículo no ahondará en la naturaleza química de los BCs, de modo que, para resumir, técnicamente se entiende que la bioenergía es aquella energía obtenida a partir de material biológico o biomasa, por lo que se considera renovable. El término biomasa, a su vez, se refiere a cualquier material no fósil de origen biológico (FAO, 2008) como cultivos energéticos, silvicultura, residuos agrícolas y desperdicios orgánicos².

La biomasa es expuesta a procesos de transformación de distinta índole, en aras de la obtención de energía. El ejemplo más simple y antiguo de la

Biomasa tiene otra acepción con un matiz más ecológico: "Materia total de los seres que viven en un lugar determinado, expresada en peso por unidad de área o de volumen". (Diccionario de la real Academia de la Lengua Española, DRAE. Recuperado el 23 de marzo de 2010, de http://buscon.rae.es/drael/SrvltConsulta?TIPO_ BUS=3\&LEMA=biomasa). Sin embargo, dada la naturaleza de los argumentos expuestos en el artículo, se obviará esta segunda interpretación. bioenergía tradicional es el la combustión de leña con propósitos de calefacción o de cocción de alimentos. Otro uso es la obtención de calor a partir de la quema de biomasa, este calor se usa para generar vapor de agua y así mover turbinas, con el fin de transformar la biomasa en electricidad.

Aunque estos dos casos son ejemplos de aplicaciones bioenergéticas, no ilustran el alcance total de los BCs. En un sentido amplio, los biocombustibles se definen como aquellos combustibles sólidos, líquidos o gaseosos obtenidos directa o indirectamente a partir de material biológico, como leña, carbón, bioetanol, biodiesel, biogás - metano- o biohidrógeno. Sin embargo, la mayoría de la literatura académica reciente se refiere a los biocombustibles dentro de la bioenergía moderna -obtención indirecta-, lo que precisa aún más el concepto y resta importancia al uso de leña para cocción directa y calefacción, es decir, la bioenergía tradicional, que pese a que su uso es muy común, está entra en desuso y no requiere intervenciones tecnológicas para su aprovechamiento (IEA, 2008).

Dale (2007) afirma que la energía no se valora per se, es decir que su importancia no reside en su fuente o en el contenido energético, sino que su valor está determinado por la forma en que ésta se dispone al consumidor final. Cualquier forma de energía puede clasificarse según su procedencia, pero es más significativo categorizarla con base en su aplicación, de forma 
que sea considerado un calefactor, un abastecedor de electricidad o un proveedor de capacidad de locomoción.

Los BCs líquidos están destinados casi en exclusiva al sector transporte, las demás funciones -cocción, calefacción y electricidad- son provistas por los componentes sólido y gaseoso. Aquí radica la importancia de este energético: es muy versátil y cubre necesidades, como el transporte, que otras fuentes renovables como la energía eólica o la hidroeléctrica no alcanzarán a satisfacer en un futuro cercano.

Es posible encontrar muchas referencias recientes, en publicaciones de organismos multilaterales y la comunidad académica, en las que sus autores clasifican la definición de los BCs como aquellos combustibles líquidos usados para locomoción (Demirbas, 2007; FAO, 2008; Honty y Gudynas, 2007; Pistonesi et al., 2008). Cuando su producción sólo se basa en monocultivos extensivos se les conoce también como agrocombustibles ${ }^{3}$.

Una vez definidos los BCs, es posible clasificarlos. Esta categorización puede

Ciertos autores, que se declaran en contra de reconocer este tipo de energía como opción sostenible, prefieren evitar el uso de la palabra biocombustible, por considerar que la raíz bio otorga una idea falsa de efectos ecológicos positivos. Dichos autores proponen, en cambio, el uso del término agrocombustible, que hace explicito el uso de grandes cultivos para la producción de combustible (Honty Gudynas, 2007; Pistonesi, et al., 2008). En el presente artículo no se usa la palabra biocombustible bajo ninguno de los matices anteriores, sino que se refiere meramente al origen biológico de la materia prima. ser muy general, es decir, por su estado físico -como se resume en la figura $1-$, que a su vez determina el uso, como se explicó anteriormente. De forma específica, se puede hacer una subclasificación de los BCs en su estado líquido, dada la aproximación técnica que los define y que determina el tipo de materia prima y la tecnología requerida para producirlos (ver figura 2).

Por ahora, para la primera clasificación se trabajará con la definición de BCs en su máxima amplitud, a la luz de bioenergía moderna. En la figura 1 se muestra someramente el proceso de transformación desde biomasa hasta la obtención de los diferentes BCs.

Para obtener BCs sólidos, cuando la biomasa proviene de fuentes no estandarizadas -ramas, estiércol seco, leña irregular, cortezas de diversos tamaños-, se debe transformar en partículas más pequeñas, como astillas uniformes, que después puedan ser comprimidas en un molde y presentadas en formas de pellets o briquetas. Esto facilita su almacenamiento, comercialización y posterior aprovechamiento.

También es posible aplicar un proceso de tostión, similar al del café, llamado torrefacción, en el que se somete la biomasa a temperaturas entre $\operatorname{los} 200^{\circ} \mathrm{C}$ y $320^{\circ} \mathrm{C}$, en ausencia de oxígeno. Se obtienen dos productos básicos de este proceso: uno es el material sólido carbonizado, conocido como biocarbón -o biochar en inglés-, usado como combustible bajo la 
Figura 1. Clasificación de los biocombustibles según su estado físico: de la materia prima al producto

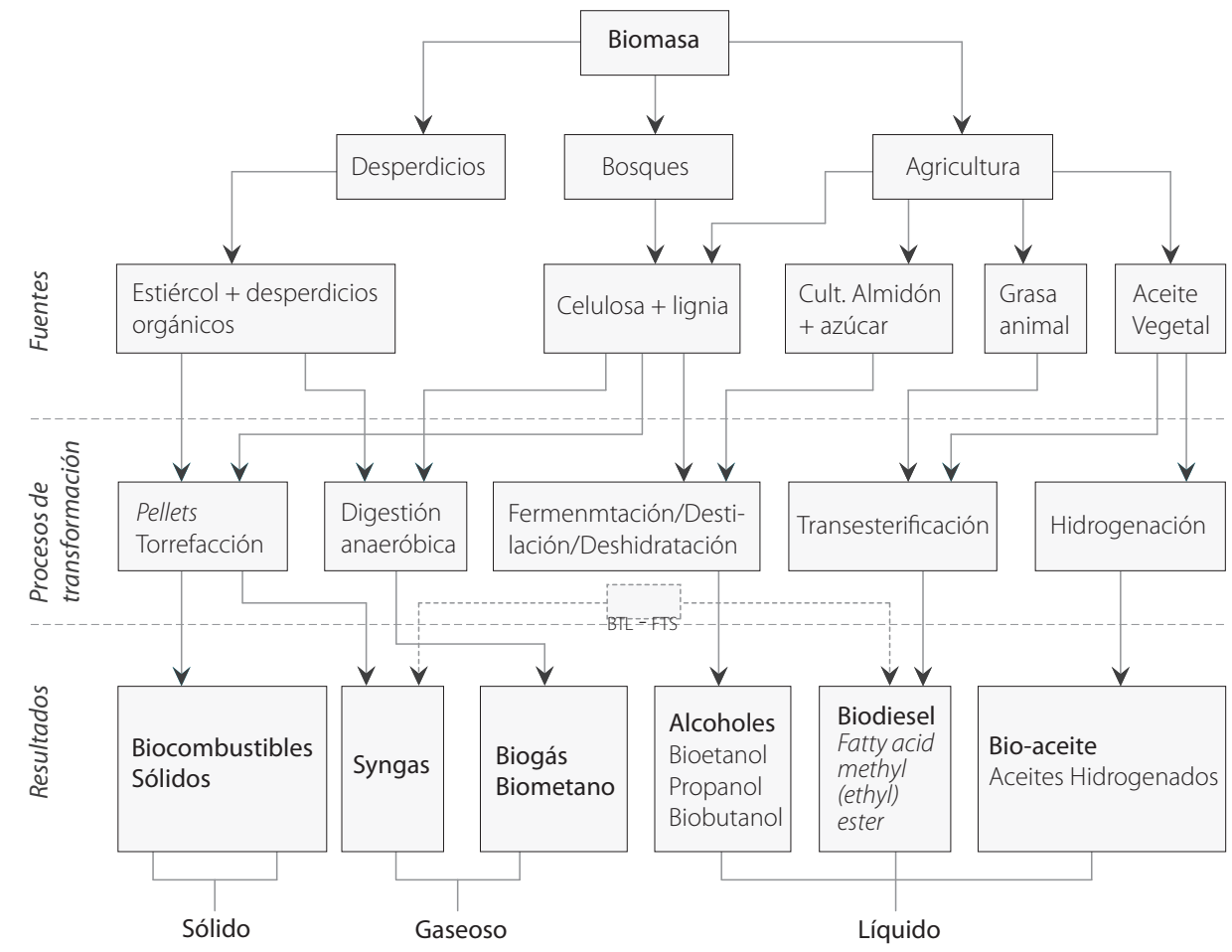

denominación de bioenergía tradicional, una contribución al manejo de desperaunque con mayor concentración o densi- dicios agrícolas, aunque también existe la dad energética (Bergman \& Kiel, 2005). posibilidad de tener cultivos que no sólo

Esta técnica puede combinarse con capturan carbono a través de su proceso la descrita anteriormente y facilita el fotosintético, sino que también "secuesproceso de elaboración de los pellets. No tran" el carbono emitido por el suelo, lo obstante, su verdadero impacto surge que genera cultivos con emisiones de carcuando es usado como agroinsumo -y no bono negativas (Mathews, 2008) ${ }^{4}$. como combustible-, durante la etapa de arado y preparación de la tierra ya que, al mezclarla con ésta, captura enormes cantidades de carbono contenido en ella y que de otra forma sería liberado a la atmósfera, lo que reduce la contribución de gases de efecto invernadero por parte de la agricultura. Así, esta práctica implica

Cuando son quemados, los BCs liberan carbono a la atmósfera para liberar su energía; sin embargo, si los cultivos necesarios para crearlos son capaces de capturar el mismo monto de carbono liberado, se entiende que su efecto es neutral; y si, como en el caso del biochar, se atrapa más carbono del que se libera, el balance es negativo. 
El segundo вс proveniente de la pirólisis es gaseoso y se denomina gas de síntesis o syngas. Se usa directamente como combustible o como producto intermedio, a través de la síntesis de Fisher-Tropsch -FTS-, un proceso químico aplicado para la elaboración de gasolina convencional, diesel y otros compuestos; este producto intermedio se emplea en la elaboración de biodiesel, metano o dimetiléter (Demirbas, 2007).

Otra forma de aprovechar la biomasa en estado gaseoso es la elaboración de gas metano o biogás mediante el almacenamiento de desechos agrícolas en un biodigestor, en el que se someten a digestión aeróbica o biodegradación, proceso del que resulta la acumulación del gas, que puede ser usado con fines de calefacción y provisión de electricidad mediante turbinas. También existe un subproducto sólido puede ser usado como combustible, sin embargo, en la mayoría de los casos, es comercializado como fertilizante.

Una fuente importante de biogás, aunque no muy limpia ni homogénea, son los rellenos sanitarios o botaderos a cielo abierto, por eso el uso de biodigestores familiares de bajo costo tienen cada vez más difusión y presentan una alternativa para poblaciones que carecen o presentan difícil acceso a la malla energética principal de un país.

Los exponentes del componente líquido en los BCs son alcoholes y aceites, los más conocidos son el bioetanol y el biodiesel.
Los alcoholes -etanol, butanol, propanol- se usan para sustituir o complementar, según sea el propósito de la política energética vigente, el uso de gasolina. Éstos se originan de productos ricos en almidón, como la papa, el maíz, la yuca o el trigo; no obstante, es posible extraerlos de diversas fuentes de azúcar como la remolacha, o su principal exponente alrededor del mundo: la caña azucarera.

Sin embargo, una tecnología más novedosa para la producción de alcohol, como se explica posteriormente, usa productos con grandes contenidos de lignina y celulosa, que constituyen una materia prima más abundante. Estas dos sustancias se acumulan en la corteza de todas las plantas, y suelen usarse comercialmente para la elaboración de papel y cartón.

Además presentan un potencial impresionante para la industria bioenergética, pues su implementación permitiría la inclusión de otros cultivos como parte de la estrategia energética -variedades de pastos, denominados gramíneas perennes, de bajo impacto ambiental y alto rendimiento-, y daría lugar al aprovechamiento de los desperdicios de diversas industrias -como la maderera, con ramas y cortezas no aprovechadas; la de procesadores de alimentos, con cascarillas de semillas producto de la tri1la; la recicladora, con papel y cartón-. Desafortunadamente, esta tecnología aún se encuentra en desarrollo y no está 
disponible a escala comercial debido a sus costos, que obedecen a la complejidad técnica implicada en el proceso.

Los aceites, por su parte, complementan o substituyen las funciones del petrodiesel, conocido en Colombia como ACPM -Aceite Combustible Para Motores-. Su materia prima proviene de fuentes animales -excepto el sebo, subproducto de la industria cárnica- $\mathrm{y}$ vegetales: semillas oleaginosas, como las de soya, las de la colza o canola, la mostaza, las de girasol o las de palma de aceite. Estos aceites son sometidos directamente a la transesterificación, un proceso en el que éstos se mezclan con un alcohol -metanol, por lo general- para romper los triglicéridos contenidos en los aceites o las grasas, y obtener biodisel -O FAME ${ }^{5}$, su nombre técnico- y glicerina, una materia prima de uso frecuente en la industria farmacéutica.

También es posible emplear aceites vegetales hidrogenados, que fueron usados para frituras o para engorde de animales, aunque la experiencia comercial de este segundo método no es tan amplia como la del fame (Evans, 2007) y los resultados son menos homogéneos, lo que dificulta garantizar la calidad del producto. Es posible usar aceites no comestibles o de baja calidad, esto

FAME, por sus siglas en inglés: Fatty Acid Methyl Ester, es la reacción catalítica entre ácidos grasos y metanol. permite la inclusión de nuevas variedades de plantas como la exitosa Jatropha curcas de la India, que no requiere suelos muy fértiles, de modo que abre las posibilidades en tierras degradadas.

Ya se han clasificado los BCs según su estado físico y procesos de elaboración. Sin embargo, a partir de este momento se entenderá por $\mathrm{BC}$ aquel combustible en estado líquido destinado al sector transporte, a menos que se enuncie lo contrario. Esto es especialmente válido para la siguiente clasificación: BC según su estado técnico.

Los BC provienen, como se ha expuesto hasta ahora, de diferentes fuentes y tienen diversos niveles de complejidad con respecto a la tecnología requerida para producirlos y procesarlos. Esto permite clasificarlos en cuatro generaciones diferentes, como se presenta en la figura 2 y se explica a continuación.

Biocombustibles de primera generación $-\mathrm{BC}_{1} \mathrm{G}-$ : los $\mathrm{BC}_{\mathrm{I}} \mathrm{G}$, también llamados agrocombustibles, provienen de cultivos que usados para la alimentación humana, directa o indirectamente -como la producción de forraje para ganado-, y técnicamente son fáciles de implementar, ya que la extracción del azúcar o almidón necesario se lleva a cabo fácilmente mediante la adición de levaduras, en el caso de alcoholes, o procesos de rompimiento de la cadena de lípidos -transesterificación-, en el de los aceites. 
En esta categoría se encuentra el etanol y el butanol de caña de azúcar, maíz, papa, yuca y remolacha, así como

Figura 2. Clasificación de Biocombustibles por generaciones

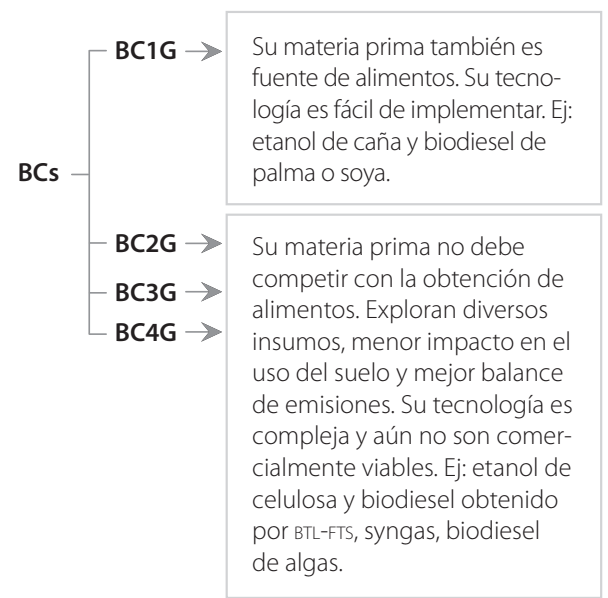

el biodiesel producido a partir de palma de aceite, soya o colza. Debido a sus relativos bajos costos, $\operatorname{los}_{\mathrm{BC}_{\mathrm{I}} \mathrm{G}}$ han sido los únicos BCs explotados comercialmente desde la primera guerra mundial.

Biocombustibles de segunda ge-

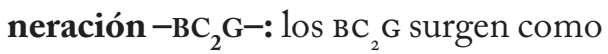
respuesta a la mayor crítica hecha a los $\mathrm{BC}_{\mathrm{I}} \mathrm{G}$ : el dilema entre comida y combustible. En el caso de fabricación de alcohol, los $\mathrm{BC}_{2} \mathrm{G}$ sólo requieren fuentes de lignocelulosa, lo que amplía el espectro de recursos disponibles en los que se sustenta la producción; esto incluye gramíneas -pastos-, árboles y desperdicios agrícolas.

Para la elaboración de biodiesel se usan plantas oleaginosas no comestibles: la Jatropha, el aceite de ricino o higuerilla y algunas variedades de arbustos, como la Pongammia pinnata y la Calophyllum inophyllum. Las fuentes de lignina también sirven, si son sometidas a pirolisis bajo fTs. Así, en este caso el reto no radica en el qué, sino en el cómo, ya que el procesamiento de los $\mathrm{BC}_{2} \mathrm{G}$ tiene dos posibles vías:

- La extracción bioquímica para la fabricación de alcoholes, proceso en el se usan enzimas o microorganismos diseñados genéticamente para romper la lignocelulosa, y así lograr la separación de los azúcares necesarios. Se obtiene etanol de celulosa.

- La extracción termoquímica de los aceites, usada en la fabricación de syngas para la posterior producción de diesel ${ }^{6}$. Esta técnica se conoce como biomass-to-liquid -BLT- (BioPact, 2007).

La investigación alrededor de los $\mathrm{BC}_{2} \mathrm{G}$ ha avanzado a tal punto que se han construido varias plantas piloto en Europa para su producción, y es el tema que genera más publicaciones en el área de bioenergía; pero a pesar del respaldo y el potencial que se le confiere en la academia, sus costos no lo hacen atractivo a gran escala, por lo que se considera que dicha tecnología estará disponible en el mercado en un lapso mayor a ocho años.

Como se mencionó anteriormente, el subproducto sólido que surge de este proceso es el biocarbón. 
Biocombustibles de tercera generación $-\mathrm{BC}_{3} \mathrm{G}-$ : Los $\mathrm{BC}_{3} \mathrm{G}$ intentan cubrir la brecha que dejaron los $\mathrm{BC}_{2} \mathrm{G}$ : la competencia por la tierra para producir los cultivos. La tierra cultivable cada vez es más escasa, y la implementación de proyectos bioenergéticos relacionados con $\mathrm{BC}_{\mathrm{I}} \mathrm{G}$ y BC ${ }_{2} \mathrm{G}$ requieren de este recurso. Así pues, se han desarrollado investigaciones marginales con el fin de explorar posibilidades de explotación de algas y cianobacterias para la elaboración de biodiesel.

Al principio, las pruebas se hicieron en agua dulce, pero dado que este es otro recurso escaso, se optó por adelantar pruebas con organismos marinos, con los que se alcanzaron altos índices de productividad -cien veces más eficiente que la palma de aceite, considerada hasta ahora el mejor insumo de $\mathrm{BC}_{1} \mathrm{G}$ para la producción de biodiesel-; sin embargo, al igual que para los $\mathrm{BC}_{2} \mathrm{G}$, los altos costos -en este caso dados por condiciones biológicas- han señalado su temporal inviabilidad (Gressel, 2008).

Biocombustibles de cuarta generación $-\mathrm{BC} 4 \mathrm{G}_{-}$: dada la escasa discusión acerca de $\operatorname{los}_{\mathrm{BC}} \mathrm{G}$, su literatura es ambigua. Por un lado, los presentan como aquellos que se caracterizan por contar con modificaciones genéticas sobre su materia prima, es decir, la alteración de variedades de eucaliptos tropicales -los convierten en grandes almacenadores de celulosa, bajos en lignina- y el alerce de Dahuria, una especie de pino propia de las estepas europeas. La principal característica de estos árboles es que son "máquinas" que capturan carbono, y esta condición permite considerarlos verdaderos combustibles con emisiones de carbono negativas. Su tecnología está asociada al biocarbón, subproducto de la torrefacción mencionado en los $\mathrm{BC}_{2} \mathrm{G}$ (BioPact, 2007).

Por otro lado, lo que Gressel presenta como $\mathrm{BC}_{4} \mathrm{G}$, es una extensión de los $\mathrm{BC}_{3} \mathrm{G}$, en la que también por modificación genética se crean algas, que sometidas a procesos bioquímicos a través de enzimas, producen biohidrógeno o bioelectricidad ${ }^{7}$.

Es probable que algunos autores agrupen los $\mathrm{BC}_{3} \mathrm{G}$ у $\mathrm{BC}_{4} \mathrm{G}$ en la categoría de los $\mathrm{BC}_{2} \mathrm{G}, \mathrm{y}$ es por eso que se ha escrito muy poco sobre éstos. Su estudio e implementación son proyectos que resultan interesantes conceptualmente, aunque requieren de la maduración, experimentación y discusión a la que ya han sido sometidos los $\mathrm{BC}_{1} \mathrm{G}$.

\section{Seguridad energética}

\section{y biocombustibles}

En la literatura académica se habla de seguridad energética y de su impacto para las políticas de desarrollo de un país (Hoogeveen \& Perlot, 2005; Kruyt et al., 2009). Entre las décadas de los setenta y ochenta, la seguridad energética fue asociada con la necesidad

\footnotetext{
A pesar de que ninguno de los dos productos es líquido, en la definición adoptada para los BCs en este aparte, ambos tienen aplicaciones potenciales en la industria del transporte.
} 
garantizar un abastecimiento continuo de crudo, para cubrir las necesidades productivas y de vivienda nacionales. Para los países autosuficientes, esto significa usar sus reservas de manera eficiente, en el ámbito doméstico y de tasas de exportación; mientras que para los importadores netos implica buscar otras fuentes de energía en aras de reducir las importaciones, y por lo tanto, la dependencia energética y los riesgos asociados ésta (IEA, 2002; Kruyt et al., 2009).

Esta definición se ha ampliado, para abarcar no sólo la energía proveniente de recursos fósiles, sino que cobija otras clases de energía y los riesgos que éstas involucran, como accidentes, terrorismo, inversión insuficiente en infraestructura y mercados con diseños inadecuados o cualquier circunstancia que reduzca el abastecimiento adecuado a precios asequibles. En resumen, la Agencia Internacional de Energía, -IEA, por sus siglas en inglés- establece que la "seguridad energética puede describirse como la disponibilidad física constante (de energía) a precios asequibles mientras se respetan criterios ambientales"(IEA, 2010).

De acuerdo a esta definición, el componente ambiental cobra cada vez más importancia. La inclusión de un filtro ambiental en el análisis revalúa la importancia del petróleo y otros combustibles fósiles, si se tiene en cuenta que éstos son la principal fuente de dióxido de carbono $-\mathrm{CO}_{2}^{-}$, el gas más abundante después del vapor de agua, que causa efecto invernadero.

La bioenergía no sólo tiene la posibilidad de ampliar la oferta energética, sino que ofrece características que no son cobijadas por otro tipo de energías renovables, como cubrir la necesidad de transporte a través los BCs y capturar carbono, si la tecnología - a partir de la segunda generación-lo permite. Cualquier otro tipo de energía alternativa -eólica, mareomotriz o fotovoltaica- sólo genera carbono neutral.

\section{Desarrollo sostenible}

\section{y biocombustibles}

La sostenibilidad o desarrollo sostenible -DS-, entendida como la "satisfacción de las necesidades de las generaciones presentes sin comprometer las posibilidades de las del futuro para atender sus propias necesidades" (Brundtland Commission, 1987) $)^{8}$ requiere del triple resultado ${ }^{9}$ interconexión de las esferas ambiental, económica y social (Ver figura 3).

El Ds, analizado desde un punto de económico, se interpreta como un mecanismo de maximización del bienestar canismo de maximización del bienestar

Traducción libre del autor del original: "development which meets the needs of the present without compromising the ability of future generations to meet their own needs" (Brundtland Commission, 1987, p. 54).

En el presente artículo, el uso de triple resultado se refiere a la presencia de los elementos básicos de la definición de sostenibilidad y no a la técnica contable-ambiental de medición denominada Triple-Bottom Line en la literatura anglosajona. 
social, de manera intertemporal. Esto implica, trabajar en un mundo dinámico, en el que las restricciones del modelo no sólo se limitan al presupuesto, sino que también las dotaciones iniciales de los agentes involucrados deben incluir activos ambientales -recursos naturales renovables y no renovables- $y$ capacidades tecnológicas. En el caso de este estudio, lo anterior se lee como la búsqueda del desarrollo de un país dado el establecimiento de las posibles fuentes y formas de aprovechamiento de energía frente a las necesidades energéticas del mismo.

Figura 3. Desarrollo sostenible

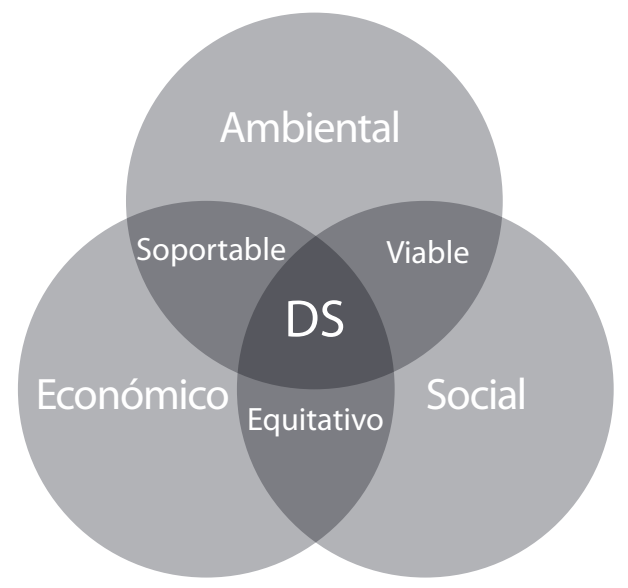

De hecho, la relación entre sostenibilidad y provisión energética es indudable, y es uno de los principales riesgos que enfrentan las naciones en vías de desarro1lo: bajo esquemas de escasa cobertura de la malla energética, el uso de formas primarias de energía como la leña-biomasa tradicional- conlleva a la degradación ambiental y a la sobrexplotación de recursos (Ölz el al, 2007; Saghir, 2006; van der Plas \& Abdel-Hamid, 2005), y los evidentes impactos negativos sobre la salud humana. Sin embargo, si se acude al otro lado del espectro, nos enfrentamos al uso de combustibles de origen fósil, que a su vez cuenta con las consecuencias ambientales adversas ya mencionadas.

En esa coyuntura surge la industria de los BCs, que desde el principio ha sido particularmente llamativa por sus potenciales resultados sostenibles $(\mathrm{Ca}-$ denas \& Cabezudo, 1998) porque:

- Es una alternativa energética "limpia" frente a los combustibles fósiles. Las emisiones de $\mathrm{CO}_{2}$ generadas por los BCs son mucho menores a las de sus equivalentes y pueden neutralizarse o incluso revertir su tendencia y capturar carbono.

- Implica desarrollo económico a escala micro, pues constituye una gran fuente de trabajo, situación que dinamiza el sector agrícola y lleva ingreso a las familias. A escala macro, representa un ahorro potencial para un país, en términos de las importaciones evitadas de petróleo y/o posibles ingresos, si dicho país es un exportador competitivo de BCs.

- Abre las puertas a sectores rurales deprimidos. El desarrollo industrial del campo va de la mano con mejoras en acceso a servicios como salud y educación. 
El desarrollo de una industria bioenergética es la oportunidad para trabajar en miras a cubrir la demanda energética de países en desarrollo, brindar posibilidades de desarrollo económico y ampliar la canasta energética de países desarrollados. Ahora bien, el alcance de los BCs es limitado y sus efectos positivos en términos de sostenibilidad, argumentos de los que se valen sus más fervorosos defensores, están aún bajo investigación académica. Esta situación ha frenado el impulso inicial de los países desarrollados y enturbia el panorama para aquellos que buscaban cambiar la petroeconomía por la bioeconomía.

En la siguiente sección se evaluará el camino recorrido por Colombia en torno a la industria de los BCs y sus efectos en torno al ds y a la seguridad energética.

\section{El caso colombiano: la disputa entre la teoría y la práctica}

En esta sección se establece un paralelo entre los aspectos normativos y positivos de la industria de los BCs. Primero se presenta un resumen teórico, que conjuga los lineamientos generales del Gobierno colombiano con los planteamientos sobre el tema a escala mundial; después, se presenta la historia de esta industria en Colombia, con el fin de establecer la convergencias entre planteamientos y resultados.

\section{Sobrevuelo por la teoría}

En la última década, la economía colombiana ha mostrado un marcado crecimiento. El producto interno bruto -PIB- ha develado cifras que superan el 4,2\% en promedio, con un pico de $7,52 \%$ en el año 2007 , uno de los comportamientos más destacados en Latinoamérica. Por lo general, este crecimiento implica procesos de modernización industrial, de urbanización y, por supuesto, de expansión de la de la demanda energética.

Si Colombia desea abonar el terreno para continuar en esta senda productiva, debe considerar la provisión de energía como una de sus variables técnicas clave. Desde esta perspectiva, el sector gubernamental -representado por el Ministerio de Agricultura- y el industrial -por la Federación Nacional de Biocombustibles- se ha aproximado al tema de los BCs, entendidos como alternativas frente a la gasolina convencional y el petrodiesel.

Elobjetivo planteado por el Gobierno nacional para llevar a cabo un plan de producción de biocombustible en el país, está dado por el aprovechamiento “...de las oportunidades de desarrollo económico y social que ofrecen los mercados emergentes de BCs, de manera competitiva y sostenible" (DNP, 2008). Pese a la exposición del objetivo, su alcance es confuso hasta cuando se hacen explícitos 
los preceptos para su puesta en marcha. De acuerdo con lo expuesto por la Unidad de Planeación Minero Energética (UPME, 2009), el propósito es velar por la diversificación de la canasta energética a través del uso de всs con criterios de:

1. Abastecimiento energético.

2. Mejoramiento de la calidad de combustibles.

3. Desarrollo agrícola.

4. Desarrollo agroindustrial.

5. Generación de empleo.

6. Sostenibilidad ambiental.

Con base en los criterios anteriores, se define la producción de biocombustible en el país como un avance en la seguridad energética mediante una alternativa basada en el desarrollo sostenible, como se explica a continuación:

Del primer criterio se entiende que el Gobierno busca la consolidación y abastecimiento de un mercado doméstico y la posible inserción en mercados externos, de manera que contribuya a la seguridad energética nacional.

El componente ambiental se enuncia explícitamente en el sexto criterio, pero también aparece implícito en el segundo, que hace referencia a la calidad: con mejoras cualitativas en los combustibles, las emisiones se reducen, además, los cultivos usados como materia prima de los BCs absorben el carbono, y el resultado de ambas condiciones es un ambiente más limpio.
El componente económico se da por el desarrollo agrícola y agroindustrial, que busca el desarrollo tecnológico y avances en la producción de la materia prima -plantas más resistentes y de mayor rendimiento-, para aprovechar mejor los suelos e incrementar la productividad, con el fin de ofrecer menores precios y mayor competitividad, lo que facilita el abastecimiento interno y la consolidación de esta industria como un modelo exportador.

Por último, el componente social -acompañado por un gran matiz económico- es la combinación entre el desarrollo agrícola y la generación de empleo, en especial si éste es de carácter rural, pues obliga a una ocupación formal de la tierra y genera un cultivo con mercado prácticamente asegurado; también inyecta capital en zonas deprimidas del país, de modo que reduce los procesos migratorios y, en consecuencia, libera presión sobre las urbes y convierte el campo en un nuevo polo de desarrollo.

Colombia presenta la industria de BCs bajo el emblema de la sostenibilidad. En la literatura académica se habla en términos similares, por lo tanto se deduce el gran sustento teórico del plan. Los tres pilares sobre los que se erige esta industria alrededor del mundo son:

- Buscar un recurso energético renovable, dadas las tensiones oca- 
sionadas por las fluctuaciones del precio del petróleo.

- Reducir el impacto de los gases de efecto invernadero, disminuyendo la provisión energética obtenida de la combustión de productos fósiles -carbón mineral, gasolina y petrodiesel-, y capturando dióxido de carbono a través del incremento de los cultivos necesarios para producir los всs.

- Diversificar las actividades agrícolas a través de un mercado nuevo, lo que conduce a la generación de oportunidades económicas para las comunidades aledañas.

Así pues, la política bioenergética nacional sigue los lineamientos generales expuestos en la comunidad internacional y, a partir de este punto, se elaboran instrumentos fiscales para la promoción de la industria. Si bien el propósito es loable, su implementación queda en entredicho: ¿está la seguridad energética nacional en riesgo?, ¿el camino recorrido hasta ahora nos lleva por una senda sostenible? En la siguiente sección se buscará atender estos interrogantes.

\section{Duro aterrizaje a la realidad}

E1 Gobierno colombiano ha visto una alternativa de desarrollo en los BCs, por eso ha puesto en marcha una serie de reglamentaciones, con el fin de facilitar el acceso a los recursos, tanto al sector privado en su conjunto, como a pequeños empresarios, para dar vía libre a este proyecto.

\section{El riesgo de la inmovilidad}

Por supuesto, uno de los argumentos expuestos aquí es la vulnerabilidad energética del país. Para ilustrar esta situación, las figuras 4, 5 y 6 muestran la composición y evolución de la canasta energética.

La oferta de energía en Colombia consiste en cinco fuentes que casi alcanzan los treinta millones de barriles equivalentes de petróleo - $\mathrm{BEP}-$ en los años 1998 y 2006. Desde el inicio del periodo analizado en la figura 4 hasta ahora, la fuente principal ha sido el petróleo, aunque presenta un declive importante desde 1998, que es aún más profundo en el 2002.

La gráfica también muestra que la provisión energética nacional está profundamente ligada al uso de bioenergía tradicional -quema de biomasa y desperdicios-, aunque a partir de 1996 su uso disminuye. La oferta de gas natural ha experimentado el crecimiento más dinámico, quizás por la conversión de parte de la flota automotriz y por la adecuación de algunas tecnologías industriales a este combustible.

El uso de la energía hidroeléctrica presentado en esta gráfica es subestimado, pues no incluye el comercio de electricidad. En la figura 5 , en cambio, el panorama se centra en la electricidad, de modo que reitera la fortaleza del recurso 
hídrico en el país, fuente responsable de casi el $80 \%$ de la provisión eléctrica para el 2007.

La figura 6 concluye que los combustibles fósiles suplen la mayoría de las necesidades energéticas colombianas, pues casi el 70\% de la energía proviene

Figura 4. Oferta primaria total de energía necesidad, por lo que es posible exportar en grandes cantidades y, en menor medida, aprovecharlo en el ámbito doméstico. Desde 1988 hasta el 2008 el consumo interno de carbón es cercano a los cinco millones de toneladas, mientras que su producción ha pasado

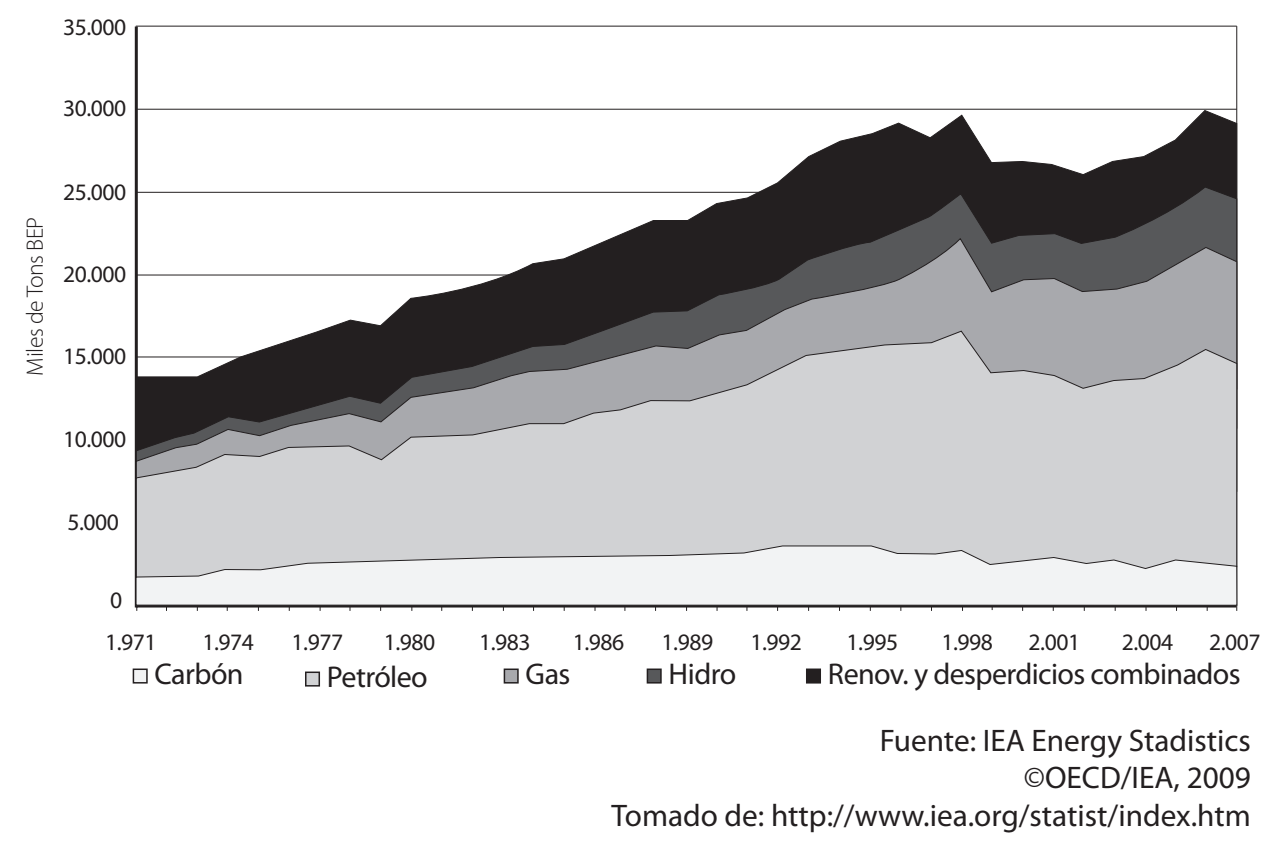

de gas, petróleo o carbón. Dado que la cobertura de electricidad del país cuenta con un respaldo hidroeléctrico importante, se entiende que la función principal de los combustibles fósiles es atender el sector transporte -mediante petróleo y gas- y demás necesidades industriales.

El carbón es un recurso abundante en Colombia y se usa para calefacción y producción eléctrica, sin embargo las hidroeléctricas nacionales cubren esta de dieciocho millones de toneladas a inicios de ese periodo a casi noventa en el 2008 (eIA, 2010).

En cambio, el petróleo sigue otra tendencia, aunque Colombia aún es autosuficiente con respecto a este hidrocarburo. En 1989 se producían un poco más de $400 \mathrm{mbd}$-miles de barriles diarios-, y la mitad se consumía al interior del país; en el 2008, el consumo aumentó tan sólo en $100 \mathrm{mbd}$ y 
Figura 5. Generación eléctrica de acuerdo al tipo de combustible, Colombia

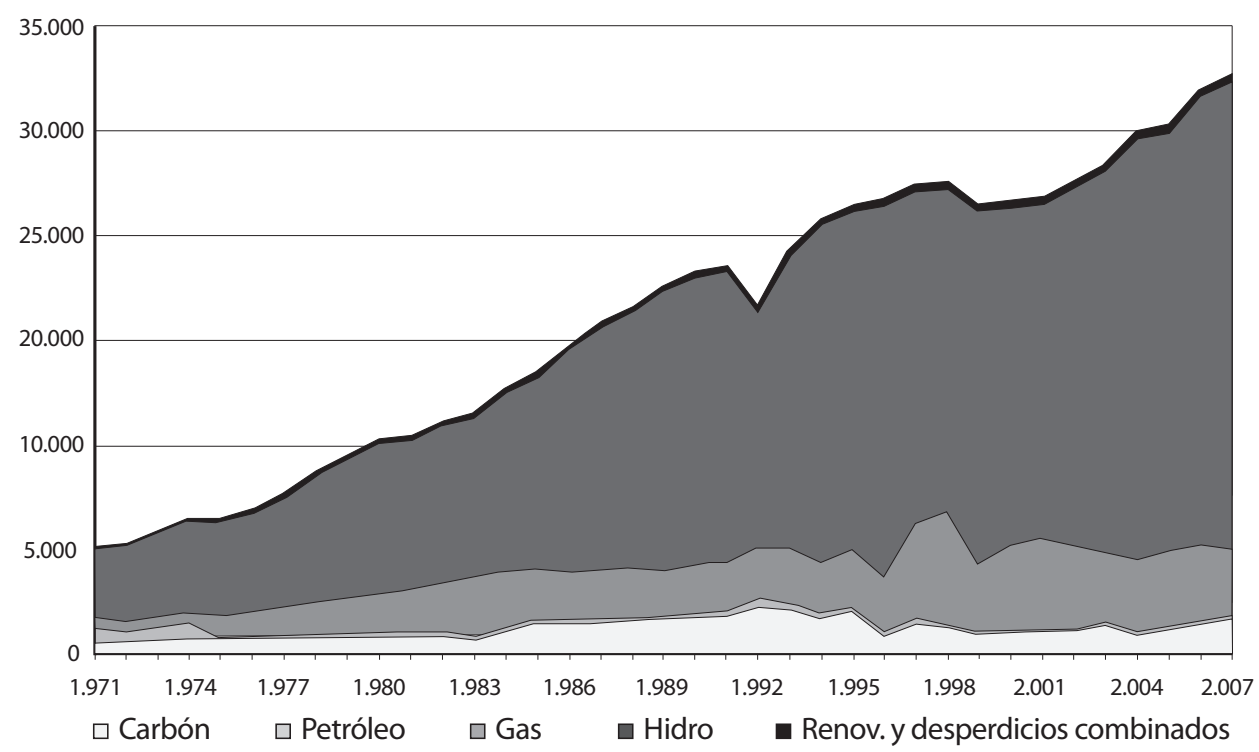

Fuente: IEA Energy Stadistics COECD/IEA Tomado de: http://www.iea.org/statist/index.htm

la producción superó los $600 \mathrm{mbd}$. La mayoría del crudo restante es exportado a Estados Unidos.

Debido a limitaciones en la capacidad de refinación algunos productos derivados, éstos deben ser importados, razón por la cual la refinería de Cartagena está en proceso de expansión desde 2009, con una inversión de 3,8 millones de dólares, para incrementar la capacidad en $165 \mathrm{mbd}$ (EIA, 2010).

A pesar de estos esfuerzos, es imposible aumentar el stock natural del recurso, y el suministro depende de los yacimientos disponibles y de la suerte en las exploraciones. Técnicamente, si se divide la cantidad de reservas de petróleo

Figura 6. Consumo total de energía en Colombia, según la fuente (2007).

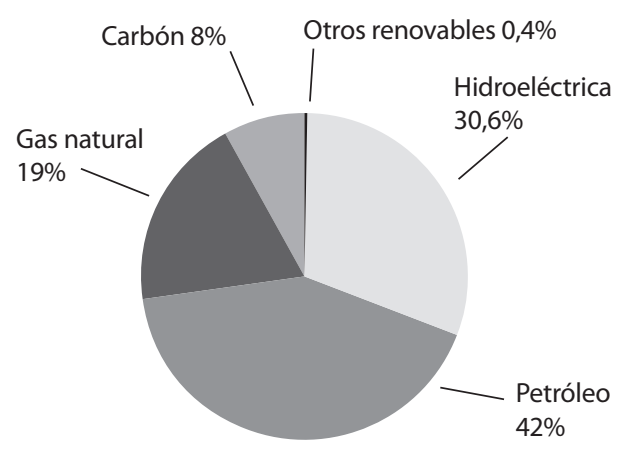

entre la tasa de explotación o producción de combustible, es posible tener una idea aproximada de cuántos años durará el recurso, esto se denomina 
relación $\mathrm{R} / \mathrm{P}$. La cifra correspondiente a Colombia en el 2008 oscila entre 6,2 -según datos de la British Petroleum (в , 2009)- y 7,8 -de acuerdo con los de la Agencia Nacional de Hidrocarburos (ANH, 2010)-, en ambos casos, es muy poco alentador.

En términos de seguridad Colombia, presenta un escenario difícil, no en toda su estructura energética, sino en la provisión de petróleo, el componente más importante de la canasta. Como las necesidades de calefacción y electricidad están casi cubiertas por otras fuentes energéticas, se concluye que es la función del petróleo como combustible para transporte la que señala una posible crisis de movilidad en el país. Esto explica la regulación de la política exportadora del hidrocarburo y la búsqueda de alternativas y/o complementos como los BCs para mitigar un posible déficit en el abastecimientos.

\section{¿Propósitos sostenibles?}

La industria de $\mathrm{BCs}$ en Colombia se sustenta en dos fuentes principales: etanol de caña de azúcar y biodiesel de palma de aceite. Existen proyectos de investigación que, en menor medida, han explorado la posibilidad de explotar remolacha azucarera y yuca como insumos principales para la elaboración de etanol. En cualquiera de estos casos, el producto se clasifica como $\mathrm{BC}_{1} \mathrm{G}$, dado que estos recursos también son fuentes de productos alimenticios.
La producción de alcohol carburante actual se concentra en el Valle del Cauca, en cinco destilerías asociadas con la producción de azúcar. Se destinan casi cuarenta mil hectáreas para la siembra de la caña y, en 2010, la capacidad instalada reportada fue cercana a los 1,1 millones de litros diarios (Fedebiocombustibles, 2010).

La demanda del combustible está asegurada desde que la gasolina mezclada fue anunciada en el $2001^{10}$ y el uso de $\mathrm{E}_{5}-95 \%$ gasolina y $5 \%$ etanolse hizo obligatorio por decreto, a partir del 2005, para ciudades con más de quinientos mil habitantes. A finales del 2009, el 80\% del país, a excepción de la

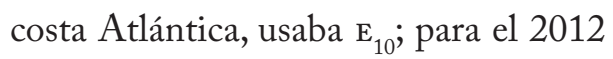
se prevé el uso de $\mathrm{E}_{15}$, aunque existen dudas sobre su conveniencia para los motores del parque automotor actual y de los requerimientos técnicos para los distribuidores (Caracol radio, 2009).

El biodiesel, por su parte, se procesa en siete plantas: una en la zona centro -Barrancabermeja-, cuatro en el norte del país -Santa Marta, Codazzi y Barranquilla- y dos en el oriente-Facatativá y San Carlos de Guaroa-. La capacidad de procesamiento total del país es de 516.000 ton/año y requiere un área aproximada de $115.000 \mathrm{Ha}$.

La demanda de este combustible también está dada por lineamientos

Ley 693 de 2001. 
gubernamentales: en el año 2004 se anunció como obligatorio el uso de la mezcla de ACPM y biodiesel ${ }^{11}$, y a partir del 2012 los vehículos importados que usen ACPM deberán estar capacitados para consumir una mezcla mínima de $20 \%$ biodiesel.

Los precios de estos BCs están dados de la siguiente manera: se garantiza el precio mínimo que represente el mejor costo de oportunidad para los productores -azúcar crudo, en el caso de etanol o aceite de palma, para el biodiesel- y de ahí en adelante se basa en los precios del mercado internacional, es decir, se pactan al precio que cueste importar el combustible equivalente.

Si bien esta regulación promueve la oferta de los BCs, garantizando oportunidades de ingreso justas para el productor, genera dos efectos negativos: por un lado, impide el acceso a combustibles más baratos al consumidor final y las mezclas obligatorias tienden a encarecer tanto la gasolina como el diesel; y por el otro, crea incentivos para desviar los insumos básicos de otras industrias, lo que amenaza la seguridad alimentaria y más aún si la industria de los BCs le apunta al mercado externo.

La globalización energética de los $\mathrm{BC}_{1} \mathrm{G}$ tiene un presente comprometido y carece de un gran futuro, las críticas que éstos reciben en términos de

Ley 939 de 2004. sostenibilidad son abundantes: generan efectos negativos en el empleo, el balance en términos de emisiones es precario, amenazan la biodiversidad y generan conflicto alrededor del uso del suelo.

Si bien los BCs son una gran fuente de empleo rural, la competitividad estimulará la búsqueda de tecnologías automatizadas, que desplacen a los encargados de la recolección de la materia prima (IDB, 2006), o bien, como ha sido reportado en Brasil, se someterá a los empleados a condiciones laborales precarias, con salarios bajos y ausencia o muy poca cobertura de servicios médicos y de seguridad industrial, que son responsabilidad del empleador (Honty y Gudynas, 2007).

La mayoría de $\mathrm{BC}_{1} \mathrm{G}$ generan emisiones positivas de gases de efecto invernadero. Es cierto que el proceso fotosintético captura el $\mathrm{CO}_{2}$, pero los cultivos extensivos y el cambio del uso del suelo liberan grandes cantidades de nitrógeno y oxígeno contenido en la tierra, que sobrepasan la capacidad de absorción de gases (BioPact, 2007).

A esto se suma el hecho de que algunas prácticas agrícolas, como el cultivo de caña requieren, dos quemas: una para eliminar la hoja y facilitar el trabajo de los corteros y otra para limpiar el suelo una vez se ha hecho el corte. En el de la palma también se realizan quemas previas al cultivo, aunque este cultivo tiene la ventaja de ser de larga duración. 
El uso extensivo de tierra y los monocultivos afectan la biodiversidad y, en consecuencia, alteran el balance ambiental. La tierra disponible en el Valle del Cauca para cultivo de caña está llegando a su límite, por lo que se propone extenderlo a los llanos Orientales, en tierras destinadas a la ganadería. Aunque esto es una solución directa al problema de la limpia de la tierra, genera una expansión indirecta en la frontera agrícola al desplazar los proyectos ganaderos ya establecidos en la zona.

La evidencia recolectada en regiones donde se han desarrollado proyectos bioenergéticos de esta naturaleza, sugiere la posibilidad de implementarlos a través de cooperativas de pequeños campesinos; pero también muestra mejores resultados, en términos de costos y homogeneidad en la provisión de la materia prima, al trabajar con grandes latifundios, lo que promueve la concentración de la tierra por parte de grandes compañías, proceso que podría generar desplazamiento y violencia, si los derechos de propiedad sobre las tierras no están bien establecidos o no se hacen respetar por parte del Estado (Cotula, et al., \& Vermeulen, 2008). Esto se agudiza cuando se presenta corrupción en la asignación de créditos blandos o auxilios agrícolas diseñados por el Estado, tal como sucedió en el programa Agro Ingreso Seguro, controlado por el Ministerio de Agricultura.

Cuando los mercados de BCs funcionan simultáneamente, incrementan el valor de los productos básicos, como sucedió en México con el efecto torti1la (Rutz et al., 2008), cuando el precio del maíz se disparó al iniciar la producción de etanol. Esto tiene un mayor impacto en la población rural de bajos recursos, pues disminuye el efecto positivo inicial; pero es aún más crítico para la población urbana más necesitada, ya que gran parte de su ingreso es destinado a la alimentación.

Las certificaciones internacionales que catalogan a los BCs como un producto sostenible, aún no se han configurado por completo, pues las tecnologías para producirlos no han madurado, pero es evidente que favorecerán los proyectos más relacionados con los $\mathrm{BC}_{2} \mathrm{G}$ y superiores.

Todos los esfuerzos científicos para aumentar productividad y reducir requerimientos agrícolas -agua, fertilizantes y calidad del suelo- son un avance para esta industria, por eso es fundamental el apoyo continuo a asociaciones como la Federación Nacional de Combustibles, Clayuca, ciat, Cenipalma y Cenicaña. Sin embargo, los esfuerzos deben apuntar a la apropiación de nuevas tecnologías e insumos para dinamizar el sector.

La industria de BCs tiene un gran potencial en Colombia, y hay razones de peso para llevarla a cabo; pero las condiciones actuales dificultan aprovechar esta oportunidad al máximo, pues crean barreras fuertes en el orden social, que hacen que éste no se considere un 
proyecto sostenible, sino viable, en términos de la figura 3 .

Es fundamental fortalecer el sistema legal en torno a un plan de ordenamiento territorial, que permita el acceso al mercado a pequeños agricultores, y diseñar planes de asignación de la tierra cuidadosos para minimizar impactos sobre el ecosistema. De lo contrario, será muy difícil que el producto obtenga la certificación en sostenibilidad, un impedimento para la inserción en los mercados internacionales.

\section{Conclusiones}

$\mathrm{La}$ industria de los BCs en Colombia ofrece una alternativa, no para todas las necesidades energéticas del país, pero sí para suplir puntualmente la urgencia en el transporte, dado que el petróleo -la principal fuente del combustible empleado para tal fin- escasea y tiene niveles de exportación altos.

Sin embargo, presenta fallas como alternativa sostenible, dado que sus dos productos principales, el etanol de caña y el biodiesel de palma, son $\mathrm{BC}_{1} \mathrm{G}$, aunque los demás estados tecnológicos $-\mathrm{BC}_{2} \mathrm{G}, \mathrm{BC}_{3} \mathrm{G}$ Y BC ${ }_{4} \mathrm{G}-$ aún no están disponibles en el mercado.

La investigación científica en torno a los BCs puede optimizar indicadores ambientales y económicos a través de mejoras agrícolas, diversificación de cultivos y disminución de agroquímicos. No obstante, su componente social depende tanto de la conciencia privada, como del monitoreo estatal, de tal manera que debe hacerse de ésta una industria incluyente, que se fundamente en la alta participación de pequeños agricultores y favorezca al consumidor final.

E1 Gobierno está decidido a apoyar la industria, de ahí la importancia de estimular la oferta y generar demanda, pero la forma y magnitud de estos auxilios debe replantearse para evitar la concentración de los beneficios en manos de unos pocos, como ha sucedido hasta ahora. Esto fortalece el carácter social de la industria, facilita su certificación sostenible y posibilita el acceso a otros mercados.

\section{Referencias}

1. Anh, Agencia Nacional de Hidrocarburos. (2010). Cifras y estadísticas. Recuperado el 7 de abril de 2010 desde http://www.anh. gov.co/es/index.php?id=8

2. Asocaña, (2009). Balance del sector Azucarero Colombiano 2000-2009. Cali, Colombia: Asocaña.

3. (2010). Estadísticas de Asocaña con indices de precios de azúcar y precio de alcohol en el mercado nacional a diciembre de 2009. Cali, Colombia: Asocaña.

4. Bergman, P.C. \& Kiel,J.H.(2005). Torrefaction for biomass upgrading. 
14th European Biomass Conference \& Exhibition. Paris, Francia. Recuperado el 5 de abril de 2010 desde http://www.ecn.n1/docs/library/report/2005/rx05180.pdf

5. BioPact, (2007). A Quick Look at 'Fourth Generation' Biofuels. Recuperado el 5 de abril de 2010 desde http://news.mongabay. com/bioenergy/2007/10/quicklook-at-fourth-generation.html

6. Bmi, Bussiness Monitor International, (2008). Colombia Power Report Q4 2008:Business Monitor International.

7. BP, British Petroleum, (2006). Quantifying Energy: BP Statistical Review of World Energy June 2006. Recuperado el 10 de marzo de 2010 desde http://www.bp.com/ liveassets/bp_internet/russia/ bp_russia_english/STAGING/ local_assets/downloads_pdfs/s/ Stat_Rev_2006_eng.pdf

8. . (2009). BP Statistical Review of World Energy June 2009. Recuperado el 10 de marzo de 2010 desde http://www.bp.com/ liveassets/bp_internet/globalbp/ globalbp_uk_english/reports_ and_publications/statistical_energy_review_2008/STAGING/ local_assets/2009_downloads/ statistical_review_of_world_energy_full_report_2009.pdf
9. Brundtland Commission.(1987). Our Common Future. Boston: Oxford University Press.

10. Cadenas, A., \& Cabezudo, S. (1998). Biofuels as Sustainable Technologies: Perspectives for Less Developed Countries. Technological Forecasting and Social Change, No. 58, pp. 83-103.

11. Caracol Radio, (2009). Colombia no está lista para usar mezcla de etanol y gasolina a partir del 2012, dicen ingenieros de Petróleo. Recuperado el 8 de abril de 2010 desde http://www.caracol.com.co/nota. aspx?id=800628

12. Chacón,J.y Gutiérrez,R.(2008, 20 de diciembre). Controversia por precio de etanol. Elespectador.com. Recuperado el 13 de marzo de 2010 desde http://www.elespectador. com/node/100690/noticias/paz/ articulo-207096-nuevo-frentede-obra-calle-26-entre-carreras57-y-71

13. Corley, R.H. (2008). How much palm oil do we need?. Environmental Science E Policy. En prensa, prueba corregida.

14. Cotula, L.; Dyer, N. \& Vermeulen, S. (2008). Bioenergy and Land Tenure: the Implications of Biofuels for Land Tenure and Land Policy: Food and Agriculture Organization of the United Nations.

15. Dale, B. (2007). Thinking Clearly about Biofuels: Ending the 
Irrelevant 'Net Energy' Debate and Developing Better Performance Metrics for Alternative Fuels. Biofuels, bioprod, bioref. No. 1, pp. 14-17.

16. Demirbas,A. (2007). Progress and Recent Trends in Biofuels. Progress in Energy and Combustion Science, No. 33, pp. 1-18.

17. dnp, Departamento Nacional de Planeación, (2008). Lineamientos de política para promover la producción sostenible de Biocombustibles en Colombia. Documento Conpes 3510.

18. Doel, K. \& Junne, G. (1986). Product Substitution Through Biotechnology: Impact on the Third World. Trends in Biotechnology, No.(4), pp. 88-90.

19. Doherty, S. \& Rydberg, T. (2002, enero). Ecosystem Properties and Principles of Living Systems as Foundation for Sustainable Agriculture: Critical Reviews of Environmental Assessment Tools, Key Findings and Questions from a Course Process. Ekologist Lantbruk, No. 32.

20. Dong, F. (2007). Food Security and Biofuels Development: the Case of China. Center for Agricultural and Rural Development. Iowa State University.

21. EIA, (2010). Colombia Energy Data, Statistics and Analysis. Recuperado el 7 de abril de 2010 desde http:// www.eia.doe.gov/cabs/Colombia/
22. Evans, G. (2007). Liquid Transport Biofuels: Technology Status Report. York: The National Non-Food Crops Centre, NnFcc.

23. FAO, Food and Agriculture Organization of the United Nations, (2008). The State of Food and Agriculture. Biofuels: Prospects, risk and opportunities. Rome, Italy: FAO.

24. Federación Nacional de Biocombustibles, (2010). Plantas productoras de etanol - Proyectos. Recuperado el 8 de abril de 2010 desde http://www.fedebiocombustibles. $\mathrm{com} / \mathrm{v} 2 /$ proyecto-web-id-18.htm

25. Fehér, A. \& LÝdia, K. (2005). An Analisys of Indicator for Sustainable Land Use Based on Research in Agricultural Landscape. Handbook of Sustainability Research, No. 20, pp. 49-67.) Ed. Walter Leal Filho. Peter Lang Publishing Group, Europäischer Verlag der Wissenschaften.

26. Frondel, M. \& Peters, J. (2007). Biodiesel: A New Oildorado? Energy Policy, No. 35 (3), pp. 1675-1684.

27. Gerpen, J.V. (2005). Biodiesel Processing and Production. Fuel Processing Technology, No. 86 (10), pp. 1097-1107.

28. Gressel, J. (2008). Transgenics are Imperative for Biofuel Crops. Plant Science, No. 174, pp. 246-263.

29. Guzmán, J.L. (2009, 29 de abril). En Colombia no modificarán el 
precio del etanol. NextFuel: Portal de información y noticias sobre Biodiesel y energías renovables. Recuperado el 17 de julio de 2009 desde http:// www.biodiesel.com.ar/?p=1300

30. Henderson, J. \& Osborne, D.J. (2000). The Oil Palm in all our Lives: How this Came About. Endeavour, No. 24 (2), pp. 63-68.

31. Hill, J.; Nelson, E.; Polasky, S. \& Tiffany, D. (2006). Environmental, Economic, and Energetic Costs and Benefits of Biodiesel and Ethanol Biofuels. Proceedings of the National Academy od Sicences of the USA, No. 103 (30), 11206-11210.

32. Honty, G. y Gudynas, E. (2007). Agrocombustibles y desarrollo sostenible en América Latina y el Caribe. Montevideo: Observatorio de Desarrollo.

33. Hoogeveen, F. \& Perlot, W. (2005). Tomorrow's Mores: The International System, Geopolitical Changes and Energy: Groningen, Clingendael International Energy Programme.

34. idb, Inter-American DevelopMENT BANK, (1990). Washington: IDB.

35. (2006). Biofuels in Latin America and the Caribbean. Canada: IDB.

36. iea, International Energy Agency, (2002). Energy Security: Working Papers: Organisation for Economic Co-operation and Development, OeCD.
37.

Outlook. Paris: Oecd \& Iea.

38. (2010). Energy Security. Recuperado el 22 de marzo de 2010 desde http://www. iea.org/subjectqueries/keyresult. asp?KEYWORD_ID=4103

39. Kinjaroun, C.; Tubtimdee, C. \& Piumsomboon, P. (2009). Lca Studies Comparing Biodiesel Synthesized by Conventional and Supercritical Methanol Methods. Journal of Cleaner Production, No. 17 (2), pp. 143-153.

40. Kline, K.; Dale, V.; Lee, R. \& LeIBy, P. (2009). In Defense of Biofuels, Done Right. Issues in Science and Technology, No. 25 (3), pp.75-84.

41. Kruyt, B.; van Vuurena, D.P.; de Vriesa, H.J. \& GroenenBERG, H. (2009). Indicators for Energy Security. Energy Policy, No. 37, pp. 2166-2181.

42. Marchetti, J.M., Miguel, V.U. \& Errazu, A.F. (2007). Possible methods for Biodiesel Production. Renewable and Sustainable Energy Revierws, No. 11 (6), pp. 1300-1311.

43. Mathews, J. (2007a). Biofuels: What a Biopact between Nort and South could achieve. Energy policy, No. 35, 3550-3570.

44. . (2007b). Renewable Energies, Development Blocs and Developing Countries. Artículo 
presentado en DRUID Summer conference 2007.

45. . (2008). Carbon-Negative Biofuels. Energy Policy, No. 36, pp. 940-945.

46. \& TAN, H. (2009, 11 de noviembre). Biofuels and Indirect Land Change Effects: the Debate Continues. Society of Chemical Industry John Wiley E Sons Ltd. Biofuels, Bioprod, Bioref. No. 3 (6), pp. 575 - 576.

47. Mulugetta, Y. (2008). Evaluating the Economics of Biodiesel in Africa. Renewable and Sustainable Energy Reviews. En prensa, prueba corregida.

48. Ölz, S.; Sims, R. \& Kirchner, NICOLAI (2007). Contribution of Renewables to Energy Security. Francia: OECD \& IEA.

49. Oxford Analytica. (2007, 2 de febrero). Bush Outlines '20 In 10' Energy Plan. Forbes.com. Recuperado el 20 de marzo de 2010 desde http://www.forbes. com/2007/02/01/bush-energyoil-biz-cx_0202oxford.html

50. Pistonesi, H.; Nadal, G.; Bravo, V. \& Bouille, D. (2008). The Contribution of Biofuels to the Sustainability of Development in Latin America and the Caribbean. Santiago de Chile: Cepal, Gtz \& Bundesministerium für wirtschaftliche Zusammenarbeit und Entwicklung.
51. Reijnders,L.\&Hujbregts,M.A. (2008). Palm Oil and the Emission of Carbon-Based Greenhouse Gases. Journal of Cleaner Production, No. 16 (4), pp. 477-482.

52. Rutz, D.; Janssen, R.; Anton, H.; Helm, P.; Rogat, J.; Hodes, K. et al. (2008). Biofuels assesment on technical opportunities and research needs for Latin America: BioTop. Munich: Biofuels RTD-cooperation Latin America-Europe.

53. SAghir, J. (2006). Global Energy Security Perspectives. Artículo presentado en E7 Summit Meeting 2006.

54. Smeets, E. (2008). Possibilities and Limitations for Sustainable Bioenergy Production Systems. Netherlands: Utrecht University.

55. ; FAAIJ, A. \& LeWANDowski, I. (2004). A Quickscan of Global Bio-Energy Potentials to 2050. Netherland: Copernicus Institute.

56. Sustainable Production of Biomass. (2007). Testing framework for sustainable biomass. Recuperado el 3 de mayo de 2010 desde http://www.senternovem. $\mathrm{nl} / \mathrm{mm}$ files/Final\%20Report $\% 20$ from $\% 20$ the $\% 20$ project $\% 20$ group $\% 20$ Sustainable\%20production\%20 of\%20biomass\%20 February\%202007\%20-\%20Testing\%20framework\%20for\%20 sustainable\%20biomass_tcm24222027.pdf 
57. Un-Energy, (2007). Sustainable Bioenergy: A Framework for Decision Makers. New York: United Nations.

58. upme, Unidad de Planeación Minero Energética. (2009). Documento "Biocombustibles en Colombia”.

59. van der Plas, R.J. \& ABdeLHamid, M.A. (2005). Can the Woodfuel Supply in sub-Saharan
Africa be Sustainable? The case of N'Djaména, Chad,. Energy Policy, No. 33, pp 297-306.

60. van Dyne, D.L.; Weber, J.A. \& Braschler, C.H. (1996). Macroeconomic Effects of a Community-Based Biodiesel Production System. Bioresource Technology, No. 56 (1), pp. 1-6. 